\title{
The Spanish strategy for nutrition, physical activity and the prevention of obesity
}

\author{
Maria Neira ${ }^{1 *}$ and Mercedes de Onis $^{2}$ \\ ${ }^{1}$ Spanish Food Safety Agency, Ministry of Health and Consumer Affairs, Madrid, Spain \\ ${ }^{2}$ Department of Nutrition, WHO, Geneva, Switzerland
}

\begin{abstract}
The prevalence and increasing trends of obesity in Spain are a matter of concern. In adults, the prevalence of obesity and overweight is 14.5 and $38.5 \%$, respectively, whereas in children and adolescents it is 13.9 and $26.3 \%$. This situation prompted the Spanish Ministry of Health and Consumer Affairs to draw up the Strategy for Nutrition, Physical Activity and the Prevention of Obesity (NAOS), which aims to improve the diet and encourage the regular practice of physical activity by all citizens, with special emphasis on children. Coordinated by the Spanish Food Safety Agency and the General Directorate of Public Health, a wide range of stakeholders participated in drafting the Strategy through a broad consultative process. Anchored on the core goal of adopting a lifelong perspective in the prevention and control of obesity, NAOS encompasses recommendations for action in four fields: family and community, schools, private sector, and the health system. Launched on 10 February 2005, the Strategy will undergo careful monitoring and evaluation. A newly created Obesity Observatory will carry out epidemiological surveillance, define indicators to measure impact of interventions, facilitate the exchange of experiences among different initiatives, identify research priorities, monitor adherence and application of the self-regulation agreements, and conduct rigorous evaluation of initiatives to identify those that are successful and should be prioritized. To our knowledge, NAOS is the first strategy of its kind in Europe and we recommend it as an example to be followed by countries that, like Spain, face the challenge of combating the pervasive epidemic of obesity.
\end{abstract}

Obesity: Overweight: Nutrition: Diet: Physical activity

A profound shift in the major causes of health and disease has occurred in developed countries and is under way in many developing countries. Globally, the burden of non-communicable diseases has rapidly increased (World Health Organization, 2002). The most important risk factors for these conditions include high blood pressure, high concentrations of cholesterol in the blood, inadequate intake of fruits and vegetables, overweight or obesity, physical inactivity and tobacco use (World Health Organization, 2002). Five of these factors are closely related to diet and physical activity.

Recognizing the heavy and growing burden of non-communicable diseases, the WHO initiated a broad consultative process in 2002 to develop a global strategy on diet, physical activity and health. Over a period of 2 years, Member States of WHO, organizations of the UN system, representatives of civil society and the private sector engaged in a number of consultations and round-table discussions that culminated in May 2004 with the endorsement by the World Health Assembly of the Global Strategy on Diet, Physical Activity and Health (World Health Organization, 2004).

The overall goal of the Global Strategy is to promote and protect health by guiding the development of an enabling environment for sustainable actions at individual, community, national and global levels that, when taken together, will lead to reduced disease and death rates related to unhealthy diet and physical inactivity (World Health Organization, 2004). The challenge is for countries to adapt the WHO global strategy to their social and cultural environments. The present paper describes how Spain has undertaken this strategy and developed the Spanish Strategy for Nutrition, Physical Activity and the Prevention of Obesity (NAOS).

\section{Prevalence and causes of obesity in Spain}

The prevalence of obesity and its rising trend over the last two decades has led to the term 'epidemic obesity' being consolidated in Spain. In the Spanish adult population (25-60 years), the prevalence of obesity and overweight is 14.5 and $38.5 \%$, respectively (Aranceta Bartrina et al. 2003). Obesity is more common in females $(15.7 \%)$ than in males $(13.4 \%)$, and the prevalence increases with age reaching figures of 21.6 and $33.9 \%$ in males and females over 55 years, respectively (Aranceta Bartrina et al. 2003).

More worrying is the situation in the young population (2-24 years), where the prevalence of obesity is $13.9 \%$, and that of overweight $26.3 \%$ (Serra Majem \& Aranceta Bartrina, 2001). In this age group, the prevalence of obesity is higher among boys $(15.6 \%)$ than girls $(12 \%)$, and the highest prevalence $(16 \cdot 1 \%)$ is seen in prepuberty (6-12 years). In comparison with other European countries, Spain has one of the highest

\footnotetext{
Abbreviation: NAOS, Spanish Strategy for Nutrition, Physical Activity and the Prevention of Obesity.

* Corresponding author: Dr Maria Neira, fax +34 91338 0375, email pneira@msc.es
} 
prevalences of childhood obesity, comparable only to that of other Mediterranean countries such as Italy, Malta and Greece.

The fast rising trends in a genetically stable population indicates that the environment, rather than biology, is driving this epidemic by creating an 'obesogenic' atmosphere characterized by excess energy intake and a sedentary life-style (Hill et al. 2003).

Spain has undergone important sociodemographic changes in recent years that have had an impact on the dietary habits and the life-style of the population. The traditional so-called Mediterranean diet has been replaced by a higher energy density diet, with more fats (principally of animal origin) and more sugars added to foods, coupled with a decrease in the intake of complex carbohydrates and fibres. These dietary changes have been accompanied by changes in life-styles leading to a reduction in physical activity both at work and during leisure time.

A balanced and healthy diet is important at all stages of life, but more so during childhood. The diet of Spanish children and adolescents is characterized by an excess of meats, cured meats, milk products and high-energy foods, including manufactured cakes and fizzy drinks (rich in fats and refined sugars, respectively), with a low intake of fruit, vegetables and cereals.

Another driving factor of the increasing trend in obesity is the physical inactivity resulting from sedentary life-styles. In children and adolescents, the number of hours spent playing on computers and video games has increased while physical exercise in everyday activities (e.g. walking to school, playing outside) has been reduced. A recent study shows that Spanish children spend on average $2.5 \mathrm{~h}$ a day watching television and a further half hour playing with video games or connected to the Internet (Asociación para la Investigación de Medios de Comunicación, 2004). Profound knowledge of all these determinants will be crucial to address the challenge of modifying the risk factors leading to obesity.

\section{Spanish Strategy for Nutrition, Physical Activity and the Prevention of Obesity}

The situation described earlier prompted the Spanish Ministry of Health and Consumer Affairs to develop NAOS (Ministry of Health and Consumer Affairs, 2004), which aims to improve the diet and encourage the regular practice of physical activity by all citizens, with special emphasis on children and adolescents. NAOS responds to WHO's request for Member States to adapt the Global Strategy (World Health Organization, 2004) to their social and cultural environments.

The multifactorial nature of the problem called for the participation of a wide range of stakeholders in drafting NAOS. Coordinated by the Spanish Food Safety Agency and the General Directorate of Public Health, representatives of different sectors took part in a broad consultative process over a period of 12 months. These included the national and regional Governments (e.g. Ministry of Education and Science; Ministry of Agriculture, Fisheries and Food; Autonomous Communities; Town Councils); the private sector (e.g. food, sports, leisure and entertainment, catering, restaurant chains); independent experts; consumer associations; medical societies and the media. In total, over 80 organizations, including universities, professional colleges, scientific institutions, foundations and associations, have cooperated in the development of the Strategy.

Anchored on the overall goal of adopting a lifelong perspective in the prevention and control of obesity, the main objectives of NAOS are:

- To promote policies and plans of action aimed at improving dietary habits and increasing physical activity in the population.

- To raise awareness through information and media campaigns about the positive impact on health of a balanced diet and regular physical activity.

- To promote nutritional education at home, at school and in the community.

- To stimulate the practice of regular physical activity in the population, with special emphasis on schools.

- To establish a framework of collaboration with the food industry to promote the production and distribution of products which contribute to a healthier and more balanced diet.

- To make professionals in the National Health System more aware in order to foster the systematic detection of obesity and problems of overweight in the population.

- To monitor the proposed measures and evaluate the results obtained as a consequence of the Strategy.

\section{Areas of intervention}

The implementation of the Strategy requires the participation of all sectors of society. Few public health undertakings have such a multisectorial and multidisciplinary involvement as that required for the implementation of this strategy. In accordance with this philosophy, the Strategy includes recommendations for action in four main sectors: the family and community, the schools, the private sector, and the health system.

\section{Family and community}

At the family and community level, action focuses on information and media campaigns, and the production and distribution of materials (e.g. dietary guidelines) aimed at promoting improved eating habits and active life-styles. The participation of professionals (e.g. sports men and women), parents and consumer associations will be sought in the search for a multiplier effect to help spread information about healthy nutrition and physical activity among children and adolescents. Similarly, cartoons and other symbols of the leisure and entertainment industry will be used to promote a positive image of active life-styles and healthy diets. The Strategy also stresses the importance of creating green areas and sports facilities in neighbourhoods to promote the regular practice of physical activity.

For diet, guidelines for populations and individuals are shown in Table 1. For physical activity, it is recommended that individuals engage in adequate levels throughout their lives. Different types and amounts of physical activity are required for different health outcomes, with a minimum of at least $30 \mathrm{~min}$ of regular, moderate-intensity physical activity (e.g. fast walking) on most days. More activity will likely be required for weight control. 
Table 1. Dietary guidelines for populations and individuals

\begin{tabular}{l}
\hline The greater the variety of food in the diet, the greater the \\
guarantee that the diet is balanced and contains all the \\
necessary nutrients. \\
Cereals (e.g. bread, pasta, rice), potatoes and pulses should \\
be the base of any diet, with the result that carbohydrates \\
represent between 50 and $60 \%$ of the energy in the diet. \\
Fats should not exceed $30 \%$ of the daily intake, the consumption \\
of saturated fats and trans-fatty acids should be reduced. \\
Proteins should provide between 10 and $15 \%$ of the \\
total energy, combining proteins of animal origin with \\
those of vegetable origin. \\
The daily intake of fruit and vegetables should be increased to \\
reach at least 400 g/day, that is, at least five portions a \\
day of these foods. \\
The consumption of products rich in free sugars, such as \\
sweets, cakes and soft drinks, should be moderated. \\
The consumption of salt, from all sources, should be reduced \\
to below 5 g/day, and the use of iodized salt promoted. \\
Drink between one and two litres of water a day. \\
Never go without a full breakfast, consisting of dairy products, \\
cereals (e.g. bread, biscuits, breakfast cereals) and fruit, \\
giving between 15 and 20 min to the meal. In this way, \\
the need to consume less nutritious food at mid-morning \\
is avoided or lessened, and physical and mental \\
performance at school is improved. \\
Involve all members of the family in activities related to eating: \\
shopping, planning the weekly menu, and preparing and \\
cooking the food.
\end{tabular}

\section{Schools}

Schools influence the lives of most children and offer numerous opportunities for teaching children about healthy diets and physical activity. School policies and programmes should support the adoption of healthy diets and physical activity by including in the academic curriculum knowledge and skills related to diet and nutrition, having appropriate facilities and equipment to promote the frequent practice of physical activity and sports, setting standards for menus served in the dining rooms, and looking after the products offered by vending machines, as well as where the machines are located and the type of advertising they carry.

\section{Private sector}

The private sector is a significant player in promoting healthy diets and physical activity. The food industry, hotel and catering companies, bakeries, sporting-goods manufacturers and advertising and recreation businesses, all have important roles to play as advocates for healthy life-styles. Voluntary agreements were signed between the Ministry of Health and Consumer Affairs and the relevant food and catering industry in order to get the Strategy underway. The commitments by the private sector include:

- Promoting healthy diets and physical activity in accordance with the overall aims of the Strategy and international standards.

- Limiting the levels of saturated fats, trans-fatty acids, free sugars and salt in existing products.

- Considering introducing new products with better nutritional values.

- Continuing to develop and provide affordable, healthy and nutritious choices to consumers.
- Providing consumers with adequate and understandable product and nutrition information.

- Practising responsible marketing that supports the Strategy, particularly with regard to the promotion and marketing of foods high in saturated fats, trans-fatty acids, free sugars or salt.

- Issuing simple, clear and consistent food labels and evidence-based health claims that will help consumers make informed choices.

- Assisting in developing and implementing physical activity programmes.

\section{Health system}

Lastly, actions in the health system strengthen the leading role to be played by paediatricians and other health staff in the prevention and early recognition of excessive weight gain. Of vital importance is to ensure that staff working in Primary Health Care are aware of the importance of overweight and obesity as a chronic condition that should receive the same attention as other non-communicable diseases, such as diabetes or high blood pressure. To this end, the systematic detection of overweight and obesity using BMI should be part of the general check-up of all patients in all specialties. In children and adolescents, BMI-for-age should be interpreted using appropriate reference data (de Onis, 2004), and patients at risk tracked and monitored. Paediatricians should promote and protect breast-feeding, and encourage parents and caregivers to promote healthy eating patterns and regular physical activity (American Academy of Pediatrics, 2003).

\section{Evaluation and monitoring}

Launched on 10 February 2005, the Strategy will undergo careful monitoring and evaluation. An Obesity Observatory of new creation will carry out epidemiological surveillance, define indicators to measure impact of interventions, facilitate the exchange of experiences among different initiatives, identify research priorities, monitor adherence and application of the self-regulatory agreements, and conduct rigorous evaluation of initiatives to identify those that are successful and should be given priority.

\section{Conclusions}

The rapid increase in non-communicable diseases witnessed in Spain in the last two decades has called for action on the part of the health authorities. The Spanish Strategy for Nutrition, Physical Activity and the Prevention of Obesity was developed to address this need. Focusing on two of the main risk factors for non-communicable diseases, the Strategy should serve as a platform from which to encourage all those initiatives which contribute to achieving the necessary social change in the promotion of a healthy diet and the prevention of a sedentary life-style.

Various sectors of society have participated in the drafting of the Strategy and all of them will be involved in its implementation. The Strategy consists of a set of actions directed at the 
entire population, but places priority on the prevention of childhood and adolescent obesity. It also has a positive spirit. The promotion of a healthy diet and an active life-style must not be tied to a repressive campaign, interpreted by citizens as being prescriptive and based on restrictions. It is not about good or bad food, but rather about a well- or poorly-balanced diet. Nor should we forget that healthy eating is compatible with pleasure and the social role that food plays in the Spanish culture.

To our knowledge, NAOS is the first Strategy of its kind in Europe and we recommend it as an example to be replicated by other countries that, like Spain, face the challenge of combating the pervasive epidemic of obesity.

\section{Acknowledgements}

We are grateful to A. Charro, B. Moreno, C. Vázquez, C. de Teresa, R. Tojo, M. Serrano, M. A. Rubio, J. Soler, G. Morande, L. Serra, J. Aranceta, F. Casanueva, M. A. Royo, J. M. Martin-Moreno, J. I. Arranz, H. Alonso and J. M. Ballesteros.

\section{References}

American Academy of Pediatrics (2003) Prevention of pediatric overweight and obesity. Policy statement. Pediatrics 112, 424-430.

Aranceta Bartrina J, Pérez-Rodrigo C, Serra-Majem L, et al. (2003) Prevalencia de obesidad en España: Estudio SEEDO 2000. Med Clin (Barc) 120, 608-612. Asociación para la Investigación de Medios de Comunicación (2004) Estudio de Audiencia Infantil. Madrid: AIMC.

de Onis M (2004) The use of anthropometry in the prevention of childhood overweight and obesity. Int $J$ Obes Relat Metab Disord 28, S81-S85.

Hill JO, Wyatt HR, Reed GW \& Peters JC (2003) Obesity and the environment: where do we go from here? Science 299, $853-855$.

Ministry of Health and Consumer Affairs (2004) Spanish Strategy for Nutrition, Physical Activity and the Prevention of Obesity (NAOS). Madrid: Ministry of Health and Consumer Affairs.

Serra Majem L \& Aranceta Bartrina J (eds) (2001) Obesidad Infantil y Juvenil. Estudio enKid (1998-2000). Barcelona: Masson.

World Health Organization (2002) Reducing Risks, Promoting Healthy Life. World Health Report. Geneva: WHO.

World Health Organization (2004) Global Strategy on Diet, Physical Activity and Health. Resolution WHA57.17. Geneva: WHO. 